\title{
Considerations on Set-Point Weight choice for 2-DoF PID Controllers
}

\author{
Víctor M. Alfaro* Ramon Vilanova ${ }^{* *}$ Orlando Arrieta ${ }^{*, * *}$ \\ * Departamento de Automática, Escuela de Ingeniería Eléctrica \\ Universidad de Costa Rica, San José, 11501-2060 Costa Rica. \\ e-mail: \{Victor.Alfaro,Orlando.Arrieta\}@ucr.ac.cr \\ ** Departament de Telecomunicació $i$ d'Enginyeria de Sistemes, ETSE \\ Universitat Autònoma de Barcelona, 08193 Bellaterra, Barcelona, \\ Spain.e-mail: \{Ramon.Vilanova, Orlando.Arrieta\}@uab.cat
}

\begin{abstract}
This paper's aim is to present an analysis of the influence of the 2-DoF controllers proportional set-point weight over the servo-control performance and to show that the removal of the existing constraint for its selection $(0 \leq \beta \leq 1.0)$ will allow to improve its performance when a high robust regulatory control system is required. A concrete analysis is conducted by using 2-DoF PID tuning approaches that explicitly take the desired robustness level as a design parameter. It is seen that as the desired robustness increases the tuning methods suggest values $\beta>1$.0. Performance looses are evaluated if we are to be constrained to the case $\beta \leq 1.0$.
\end{abstract}

Keywords: PID Control, Two-Degree-of-Freedom, Set-Point Weights

\section{INTRODUCTION}

Since their introduction in 1940 (Babb, 1990; Bennett, 2000) commercial Proportional - Integrative - Derivative (PID) controllers have been with no doubt the most extensive option found on industrial control applications. Their success is mainly due to its simple structure and meaning of the corresponding three parameters. This fact makes PID control easier to understand by the control engineers than other most advanced control techniques.

With regard to the design and tuning of PID controllers, there are many methods that can be found in the literature over the last sixty years. Special attention is made of the IFAC workshop PID'00 Past, Present and Future of PID Control held in Terrassa, Spain, on April 2000 where a glimpse of the state-of-the-art on PID control was provided. It can be seen that most of them are concerned with feedback controllers which are tuned either with a view to the rejection of disturbances (Cohen and Coon, 1953; López et al., 1967; Ziegler and Nichols, 1942) or for a well-damped fast response to a step change in the controller set-point (Martin et al., 1975; Rivera et al., 1986; Rovira et al., 1969). The Two-Degree-of-Freedom (2-DoF) formulation is aimed at trying to met both objectives. This second degree of freedom is aimed at providing additional flexibility to the control system design. See for example (Araki, 1984a,b, 1985) and its characteristics revised and summarized in (Taguchi and Araki, 2000, 2002) and (Taguchi et al., 2002), as well as different tuning methods that have been formulated over the last years (Alfaro et al., 2008; Åström et al., 1992; Åström and Hägglund, 2004; Åström et al., 1998; Gorez, 2003; Hang and Cao, 1996; Hägglund and Åström, 2002; Taguchi and Araki, 2000).
This second degree of freedom is found on the presented literature as well as in commercial PID controllers under the form of the well known set-point weighting factor (usually called $\beta$ ) that ranges within $0 \leq \beta \leq 1.0$, being the main purpose of this parameter to avoid excessive proportional control action when a set-point change takes place. Therefore the use of just a fraction of the set-point.

There is however a shift of perspective with the introduction of Robustness considerations (Åström and Hägglund $(1995,2004,2006))$. As a result, less aggressive control actions are generated and smooth responses are achieved. However, if the desired level of robustness is high, step response performance can be seriously degraded. This is the analysis conducted in this paper that leads us to conclude that the use of values of $\beta$ that are beyond the constraint, are definitively needed in order to get better step response performance. The analysis is conducted by using two existing tuning rules for 2-DoF PID controllers that include, as an explicit design parameter, the desired robustness level in terms of the Maximum Sensitivity value. This allows to analyze the effect of going on increasing the desired robustness level. The suggested value for $\beta$ goes to values $\beta>1.0$ in many of the cases therefore constraining the achievable performance if we are to be limited to a maximum of $\beta=1.0$.

It is worth to notice that even the suggestion of allowing $\beta>1.0$ seems quite common sense and natural, to the knowledge of the authors it has not still been considered. In this paper this proposal is raised within the motivation of the increased use of robustness considerations on what we could call modern control design approaches. 


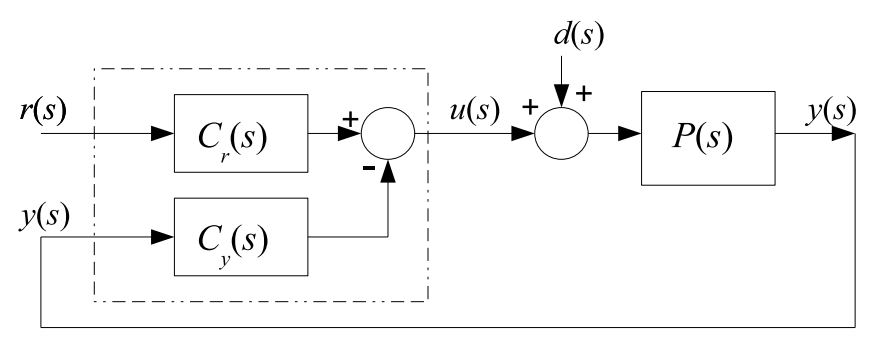

Figure 1. Closed-loop Control System

The paper is organized as follows. Section 2 introduces the control setup and the 2-DoF PID formulation. Discussion about the selection of the $\beta$ parameter is also introduced. Section 3 analyzes the effects of constraining the set-point weight on robustness based tuning rules and suggests to relax that constraint in order to met a high demanding robustness-performance tradeoff. On Section 4 an example illustrates how performance increases if larger values of $\beta$ are allowed. The paper ends in Section 5 with some conclusions.

\section{2-DOF PID FORMULATION}

Consider the closed-loop control system of Fig. 1, where $P(s)$ is the controlled process transfer function, $C_{r}(s)$ the set-point controller transfer function, $C_{y}(s)$ the feedback controller transfer function, and $r(s)$ the set-point, $d(s)$ the load-disturbance, and $y(s)$ the controlled variable (process output).

The output of the controller is given by

$$
u(s)=C_{r}(s) r(s)-C_{y}(s) y(s)
$$

Without loss of generality we will use an error feedback Ideal PID controller which equation is

$$
\begin{aligned}
u(s) & =K_{c}\left(1+\frac{1}{T_{i} s}\right) r(s) \\
& -K_{c}\left(1+\frac{1}{T_{i} s}+\frac{T_{d} s}{T_{d} / N s+1}\right) y(s)
\end{aligned}
$$

where $K_{c}$ is the controller gain, $T_{i}$ the integral time constant, $T_{d}$ the derivative time constant and $N$ the derivative filter constant (usually $N=10$ (Visioli, 2006)). Then, the controllers' transfer functions are

$$
C_{r}(s)=K_{c}\left(1+\frac{1}{T_{i} s}\right)
$$

and

$$
C_{y}(s)=K_{c}\left(1+\frac{1}{T_{i} s}+\frac{T_{d} s}{0.1 T_{d} s+1}\right)
$$

The closed-loop control system response to a change in any of its inputs, will be given by

$$
y(s)=\frac{C_{r}(s) P(s)}{1+C_{y}(s) P(s)} r(s)+\frac{P(s)}{1+C_{y}(s) P(s)} d(s)
$$

or in a compact form by

$$
y(s)=M_{y r}(s) r(s)+M_{y d}(s) d(s)
$$

where $M_{y r}(s)$ is the transfer function from set-point to controlled process variable: the servo-control closed-loop transfer function or complementary sensitivity function; $T(s)$; and $M_{y d}(s)$ is the one from load-disturbance to controlled process variable: the regulatory control closedloop transfer function or disturbance sensitivity function
$S(s)$.

Since all parameters of $C_{r}(s)$ are identical to the ones of $C_{y}(s)$ it is not possible to specify the dynamic performance of the control system to set-point changes, independently of the performance to load-disturbances changes.

If the degrees of freedom in control system are defined as the number of closed-loop transfer functions that may be selected independently (Horowitz, 1963), we have in this case a One-Degree-of-Freedom (1-DoF) control system.

The above constraint forces the designer to use a tuning rule developed for the specific required application (servocontrol o regulatory control) finding in the literature tuning rules for regulatory control (Cohen and Coon, 1953; López et al., 1967; Ziegler and Nichols, 1942), for servocontrol applications (Martin et al., 1975; Rivera et al., 1986; Rovira et al., 1969) or separate tuning rules for both applications (Chien et al., 1952; Kaya, 2004; Sung and Lee, 1999) only to mention a few. Alternatively the 1-DoF can be forced to operate in order to provide a balanced performance with respect to both operation modes. This is the so called implicit 2-DoF PID and has been presented in (Arrieta and Vilanova (2007b,c); Arrieta et al. (2008)). A collection of tuning methods may be found in O'Dwyer (2003).

It has been widely reported elsewhere that a control system with a controller optimized for load-disturbance rejection, normally presents high overshoots to set-point step inputs requiring a detuning with the consequential reduction in its regulatory performance. In such case, considerations about performance degradation of optimal tunings have to be taken into account (Arrieta and Vilanova (2007a)).

In order to provide additional flexibility for the control system design, a second degree of freedom was introduced into the PID algorithms in Araki (1984a,b, 1985) and its characteristics revised and summarized in Taguchi and Araki (2000, 2002) and Taguchi et al. (2002).

Consider now the PID controller equation (Åström and Hägglund, 2006)

$$
\begin{aligned}
u(s) & =K_{c}\left(\beta+\frac{1}{T_{i} s}+\frac{\gamma T_{d} s}{0.1 T_{d} s+1}\right) r(s) \\
& -K_{c}\left(1+\frac{1}{T_{i} s}+\frac{T_{d} s}{0.1 T_{s} s+1}\right) y(s)
\end{aligned}
$$

where $\beta$ and $\gamma$ are the set-point weights.

The $\gamma$ parameter is more frequently applied as a derivative mode switch (0 or 1 ) for the signal reference $r$. To avoid extreme instantaneous change in the controller output signal when a set-point step change occurs normally $\gamma$ is set to zero. In this case the new set-point controller transfer function is

$$
C_{r}(s)=K_{c}\left(\beta+\frac{1}{T_{i} s}\right)
$$

and the one for the feedback controller

$$
C_{y}(s)=K_{c}\left(1+\frac{1}{T_{i} s}+\frac{T_{d} s}{0.1 T_{d} s+1}\right)
$$

which is the same as (4) above.

In commercial controllers the proportional set-point weight $\beta$ may be selected only in the $0 \leq \beta \leq 1.0$ range.

Given a controlled process $P(s)$, the feedback controller $C_{y}(s)$ parameters $\left(K_{c}, T_{i}, T_{d}\right)$ may be selected to achieve a target performance for the regulatory control $M_{y d}(s)$, 
and then using the proportional set-point weight $(\beta)$, in the set-point controller $C_{r}(s)$, to modify the servo-control performance $M_{y r}(s)$.

Under the above degree of freedom definition, we have now a Two-Degree-of-Freedom (2-DoF) control system. This option allowed the development of sets of tuning methods for the 2-DoF controllers as the ones found in (Alfaro et al., 2008; Åström et al., 1992; Åström and Hägglund, 2004; Åström et al., 1998; Gorez, 2003; Hang and Cao, 1996; Hägglund and Åström, 2002; Taguchi and Araki, 2000).

With regard to the commercial implementation of the PID algorithms, it is usually to find that most of them are of 1DoF type like the ones described in (ABB ((n.d.); Foxboro (1998); Fuji (2001); Honeywell (2007); Rockwell (2003, 2005)) a few include a set-point filter ((Omron, 2007; Yokogawa, (n.d.)) and very few have 2-DoF capabilities ((Emerson, 2008; Mitsubishi, 2002)). In particular, the 2-DoF PID controller in (Emerson (2008)) allows both weights in (7) $(\beta$ and $\gamma)$ to be selected in the full 0 to 1 range.

\section{PROPORTIONAL SET-POINT WEIGHTING ANALYSIS}

From (5) and (6) the servo-control closed-loop transfer function is

$$
M_{y r}(s)=\frac{C_{r}(s) P(s)}{1+C_{y}(s) P(s)}
$$

and the one for the regulatory control

$$
M_{y d}(s)=\frac{P(s)}{1+C_{y}(s) P(s)}
$$

which are related by

$$
M_{y d}(s)=C_{r}(s) M_{y r}(s)
$$

Using (8) in (12) we have

$$
M_{y d}(s)=K_{c}\left(\frac{\beta T_{i} s+1}{T_{i} s}\right) M_{y r}(s)
$$

On the other hand, the characteristic polynomial of the closed-loop control system is

$$
p(s)=1+C_{y}(s) P(s)
$$

from where it can be obtained the closed-loop poles location; therefore the closed-loop stability; depends only on the $C_{y}(s)$ parameters, hence not affected by $\beta$.

This fact makes possible to design first the feedback controller considering the regulatory control performance and the closed-loop control system robustness and, on a second step to modify the set-point controller considering only the servo-control performance (by the introduction of $\beta$ ).

Although, the instant change in the controller output signal to a step set-point change is given by

$$
\Delta y_{r}=K_{c} \beta \Delta e_{r}=K_{c} \beta \Delta r
$$

Since the performance optimization of a regulatory control system requires controllers' gains higher than the optimization of the same loop for servo-control operation, the use of a proportional set-point weight $\beta<1$ allows to shift to the left the controller integral mode zero to a desired position to reduce the controlled signal overshoot and also to decrease the instant change in the controller output.

From the above presented analysis, it is clear that the use of a 2-DoF controller improves the servo-control performance and no questions arise about the manufactures imposed constraint on the proportional set-point weight selection range. It is along this framework that the above indicated tuning rules for 2-DoF PI and PID controllers; including the ones that take into consideration the control system robustness; constraint the set-point weight to $0 \leq \beta \leq 1$ respecting the allowed range in commercial controllers (see for example (Alfaro et al., 2008; Åström and Hägglund, 2004; Åström et al., 1998; Gorez, 2003; Hägglund and Åström, 2002; Taguchi and Araki, 2000)). However, within a more modern framework robustness considerations are an integral part of practically every design approach. In such cases, as it will be explicitly shown in next section, extending the allowed range for the set-point weight will be definitively needed in order to be able to improve the servo-control performance. When a highly robust control system is required due to the expected variations in the controlled process characteristics, a significant reduction in the controller gain is needed and the performance of the control-loop will decrease. The system responses to load-disturbance and set-point changes will be slower.

This situation motivates the analysis of the proportional set-point weighting effect over the control system performance when the set-point changes, using two of the available tuning rules for $2-\mathrm{DoF}$ controllers. The choice of the presented tuning rules is based on the fact that they include, as an explicit design parameter, the desired robustness level for the closed-loop control system. This setup allows for a more concrete and objective analysis. However the analysis can be easily extended to other tuning rules as the effect of getting a more robust feedback system is by sure to generate more conservative responses.

\section{$3.1 A_{2} T_{2}$ PI Controller Tuning}

The Analytical Robust Tuning for 2-DoF PI controllers $\left(A R T_{2}\right)$ follows (Alfaro et al., 2008) and is outlined here:

- Controlled Process Model:

$$
P(s)=\frac{K_{p} e^{-L s}}{T s+1}
$$

where $K_{p}$ is the process gain, $T$ is the time constant, and $L$ is the dead-time. It will be referred to $\tau_{o}=$ $L / T \leq 1.0$ as the controlled process normalized deadtime.

- Controller's Parameters: The $A R T_{2}$ tuning equations are

$$
\begin{gathered}
\kappa_{c}=K_{c} K_{p}=\frac{2 \tau_{c}-\tau_{c}^{2}+\tau_{o}}{\left(\tau_{c}+\tau_{o}\right)^{2}} \\
\tau_{i}=\frac{T_{i}}{T}=\frac{2 \tau_{c}-\tau_{c}^{2}+\tau_{o}}{1+\tau_{o}}
\end{gathered}
$$

where $\kappa_{c}$ and $\tau_{i}$ are the controller normalized parameters and $\tau_{c}=T_{c} / T$ the design parameter ( $T_{c}$ is the target regulatory control closed-loop time constant).

- Set-point Weighting: The proportional set-point weight selection criteria is

$$
\beta=\min \left\{\frac{1}{K_{c}}, \frac{\tau_{c} T}{T_{i}}, 1\right\}
$$

- Design Parameter: The design parameter $\tau_{c}$ may be selected within the range

$$
\max \left(0.50, \tau_{c \min }\right) \leq \tau_{c} \leq 1.50+0.3 \tau_{o}
$$

where $\tau_{c m i n}$ is given by 


$$
\begin{aligned}
\tau_{c m i n} & =k_{11}\left(M_{s}\right)+\left[\frac{k_{21}\left(M_{s}\right)}{k_{22}\left(M_{s}\right)}\right] \tau_{o} \\
k_{11}\left(M_{s}\right) & =1.384-1.063 M_{s}+0.262 M_{s}^{2} \\
k_{21}\left(M_{s}\right) & =-1.915+1.415 M_{s}-0.077 M_{s}^{2} \\
k_{22}\left(M_{s}\right) & =4.382-7.396 M_{s}+3.0 M_{s}^{2}
\end{aligned}
$$

allowing to design the control system with a robustness higher than the minimum required (give it by the maximum sensitivity $M_{s}$ ).

Using (20) and (21), the lower limits for the design parameter $\tau_{c}$ may be estimated. These are shown in Table 1 for robustness $1.2 \leq M_{s} \leq 2.0$ and controlled process model normalized dead-time $0.1 \leq \tau_{o} \leq 1.0$.

As it can be seen in Table 1 the lower and higher recommended limits in (20) were reached for the extreme cases (low normalized dead-time and robustness and high normalized dead-time and robustness). For the first case this means that, slow responses with high robustness system requirements will be obtained, and for the second one, that it is not possible to obtain a system with the high robustness specified.

The controller's proportional set-point weight may be obtained with (19) and they are shown in Table 2. As can be seen in this Table the existing upper limit constraint of 1.0 for $\beta$ was intentionally relaxed (bold).

According to the $A R T_{2}$ tuning rules, when the normalized dead-time is in the upper side of the range $\left(\tau_{o} \approx 1\right)$ and the required system robustness is high, the recommended proportional weight would be higher than 1.0. As it can be seen in the last column of Table 2, this is the situation for practically all values of $\tau_{o}$ when a robustness $M_{s}=1.2$ is specified. Therefore, the imposed constraint for the $\beta$ value selection, in the available commercial Two-Degree-

Table 1. Higher Close-loop Speed Allowed $\tau_{c m i n}$

\begin{tabular}{c|ccccc}
\hline & & & $M_{s}$ & & \\
$\tau_{o}$ & 2.0 & 1.8 & 1.6 & 1.4 & 1.2 \\
\hline 0.1 & 0.500 & 0.500 & 0.500 & 0.501 & 0.675 \\
0.2 & 0.500 & 0.500 & 0.500 & 0.593 & 0.864 \\
0.3 & 0.500 & 0.500 & 0.553 & 0.685 & 1.054 \\
0.4 & 0.500 & 0.513 & 0.620 & 0.777 & 1.243 \\
0.5 & 0.500 & 0.562 & 0.686 & 0.869 & 1.432 \\
0.6 & 0.535 & 0.610 & 0.753 & 0.961 & 1.622 \\
0.7 & 0.573 & 0.659 & 0.819 & 1.053 & 1.710 \\
0.8 & 0.611 & 0.707 & 0.886 & 1.145 & 1.740 \\
0.9 & 0.650 & 0.756 & 0.952 & 1.236 & 1.770 \\
1.0 & 0.688 & 0.804 & 1.019 & 1.328 & 1.800 \\
\hline
\end{tabular}

Table 2. Proportional Set-Point Weight Factor $\beta$

\begin{tabular}{c|ccccc}
\hline & & \multicolumn{4}{|c}{$M_{s}$} \\
$\tau_{o}$ & 2.0 & 1.8 & 1.6 & 1.4 & 1.2 \\
\hline 0.1 & 0.424 & 0.424 & 0.424 & 0.425 & 0.604 \\
0.2 & 0.516 & 0.516 & 0.516 & 0.608 & 0.878 \\
0.3 & 0.609 & 0.609 & 0.654 & 0.742 & $\mathbf{1 . 0 5 6}$ \\
0.4 & 0.609 & 0.618 & 0.691 & 0.806 & $\mathbf{1 . 2 9 8}$ \\
0.5 & 0.600 & 0.644 & 0.735 & 0.879 & $\mathbf{1 . 6 3 6}$ \\
0.6 & 0.619 & 0.674 & 0.783 & 0.962 & $\mathbf{2 . 1 3 8}$ \\
0.7 & 0.642 & 0.707 & 0.835 & $\mathbf{1 . 0 5 4}$ & $\mathbf{2 . 4 3 1}$ \\
0.8 & 0.667 & 0.743 & 0.892 & $\mathbf{1 . 1 5 8}$ & $\mathbf{2 . 5 0 1}$ \\
0.9 & 0.694 & 0.780 & 0.954 & $\mathbf{1 . 2 7 4}$ & $\mathbf{2 . 5 7 3}$ \\
1.0 & 0.723 & 0.820 & $\mathbf{1 . 0 1 9}$ & $\mathbf{1 . 4 0 4}$ & $\mathbf{2 . 6 4 7}$ \\
\hline
\end{tabular}

of-Freedom PID controllers, does not allow the designer to use the full capabilities of these controllers.

\subsection{Integrated Absolute Error (IAE) Optimized PID Tuning}

A tuning method for 2-DoF PI and PID controllers that optimizes their performance under a IAE cost functional ensuring at the same time a minimum closed-loop robustness $\left(M_{s}\right)$ is described in Méndez (2008). Bellow are presented the controller parameters for one particular robustness level, say $M_{s}=1.4$.

- Controlled Process Model:

$$
P(s)=\frac{K_{p} e^{-L s}}{(T s+1)(a T s+1)}
$$

where $K_{p}$ is the process gain, $T$ is the dominant time constant, $a$ is the time constants ratio and $L$ is the dead-time $\left(\tau_{o}=L / T\right)$.

- Controller's Set-Point Weight: Table 3 shows the $P I D_{2}$ controller's set-point weighting factor for the $M_{s}=1.4$ case corresponding to different values of $\tau_{o}$ and $a$. As shown in this table for this robustness most of the recommended proportional set-point weights exceed the 1.0 upper limit (bold).

Table 3. IAE- $M_{s} P I D_{2}$ Controller Set-Point Weight $\beta$

\begin{tabular}{c|ccccccc}
\hline & & & & $\tau_{o}$ & & \\
$a$ & 0.1 & 0.25 & 0.50 & 0.75 & 1.0 & 1.50 & 2.0 \\
\hline 0.25 & 0.636 & 0.819 & $\mathbf{1 . 0 7 3}$ & $\mathbf{1 . 2 5 6}$ & $\mathbf{1 . 4 1 3}$ & $\mathbf{1 . 6 6 5}$ & $\mathbf{1 . 8 3 9}$ \\
0.50 & 0.585 & 0.731 & 0.992 & $\mathbf{1 . 1 8 9}$ & $\mathbf{1 . 3 3 8}$ & $\mathbf{1 . 6 3 0}$ & $\mathbf{1 . 7 7 8}$ \\
0.75 & 0.588 & 0.695 & 0.921 & $\mathbf{1 . 1 0 4}$ & $\mathbf{1 . 2 6 1}$ & $\mathbf{1 . 5 5 0}$ & $\mathbf{1 . 7 5 5}$ \\
1.0 & 0.567 & 0.662 & 0.871 & $\mathbf{1 . 0 5 2}$ & $\mathbf{1 . 2 1 0}$ & $\mathbf{1 . 4 1 2}$ & $\mathbf{1 . 6 6 3}$ \\
\hline
\end{tabular}

\section{EXAMPLE}

This section provides an example to show the effect of the proportional set-point weighting over the servo-control system performance.

In order to have simulation results more close to industrial practice, in the example it is assumed that all variables can vary in the 0 to $100 \%$ normalized range and that in the normal operation point, the controlled variable, the setpoint and the control signal, have all values close to $70 \%$. For the tests a $20 \%$ change in set-point followed by a $10 \%$ change in load-disturbance will be used in all cases.

Performance: Performance will be evaluated for a setpoint change and under the presence of a load-disturbance. The Integrated-Absolute-Error (IAE) that is defined as

$$
J_{I A E} \doteq \int_{0}^{\infty}|r(t)-y(t)| d t
$$

and provides a measure for control system output performance.

Control input usage: On the other hand to evaluate the manipulated input usage, the total variation of the control effort $u(t)\left(T V_{u}\right)$ is computed. This value is defined, for a discrete signal as the sum of the size of its increments

$$
T V_{u} \doteq \sum_{k=1}^{\infty}\left|u_{k+1}-u_{k}\right|
$$

This quantity should be as small as possible and provides a measure of the smoothness of the control signal. Robustness: The maximum sensitivity value 


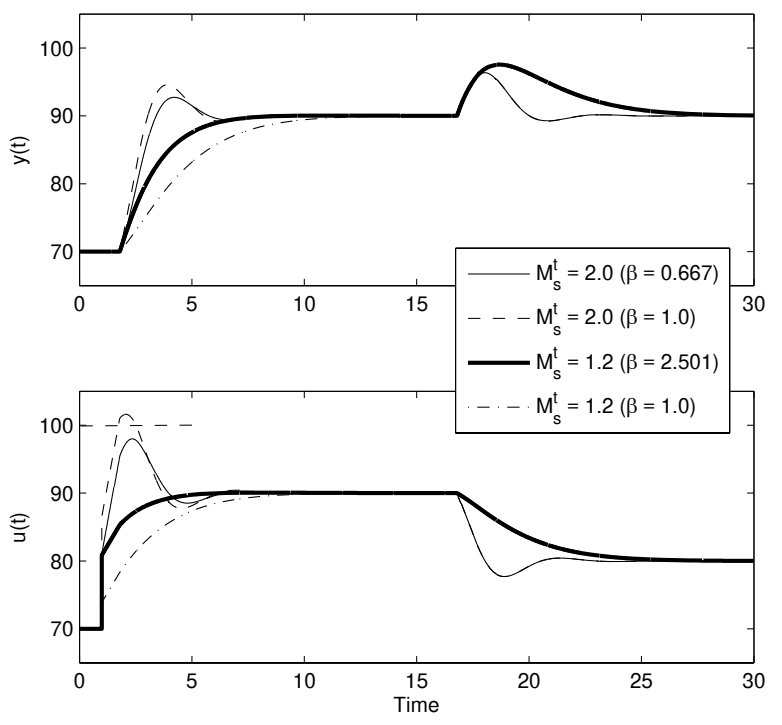

Figure 2. PI Control System Responses

Table 4. $A R T_{2}$ PI Controller Parameters

\begin{tabular}{ccccc}
\hline$M_{s}^{t}$ & $\tau_{c}$ & $K_{c}$ & $T_{i}$ & $\beta$ \\
\hline 2.0 & 0.611 & 0.828 & 0.916 & 0.667 \\
1.2 & 1.740 & 0.194 & 0.988 & 2.501 \\
\hline
\end{tabular}

$$
M_{s}=\max _{\omega}|S(\mathrm{j} \omega)|=\max _{\omega} \frac{1}{\left|1+C_{y}(\mathrm{j} \omega) P(\mathrm{j} \omega)\right|}
$$

is used as a measure of the control system robustness. Recommended values for $M_{s}$ are typically within the range $1.2-2.0$.

Consider the particular case of controlled process (16) with $K_{p}=1.0, T=1.0$ and $L=0.80\left(\tau_{o}=0.80\right)$. The $P I_{2}$ controller's parameters obtained with the $A R T_{2}$ tuning method in Section 3.1, in order to have a low robustness $\left(M_{s}^{t}=2.0\right)$ and a high robustness $\left(M_{s}^{t}=1.2\right)$ control system, are shown in Table 4 (the upper limit constraint for $\beta$ was not taken into account).

System responses are shown in Fig. 2. The figure includes also the responses obtained with $\beta=1.0$ in both cases.

The servo-control performance $\left(J_{I A E r}\right)$ and control effort smoothness $\left(T V_{u r}\right)$ as well as the obtained control system robustness $\left(M_{s}^{r}\right)$ are shown in Table 5 .

For the low robustness case $\left(M_{s}^{t}=2.0\right)$ the use of a proportional set-point weight lower than $1.0(\beta=0.667)$ allows to reduce: the servo-control controlled variable overshoot, the control effort upper value and helps to made it smoother compared with the $\beta=1.0$ case. This last case is equivalent to the use of a 1-DoF PI controller.

In the high robustness case $\left(M_{s}^{t}=1.2\right)$ the use of a proportional set-point weight higher than $1.0(\beta=2.501)$ allows to improve the servo-control performance reducing $J_{I A E r}$ without deterioration of the control effort behavior $T V_{u r}$ compared with the case of $\beta=1.0$. This is when the use of the set-point weight, in the 2-DoF PI controller, is setted to the upper limit allowed by the manufacturer.
Table 5. PI Control Performance and Robustness

\begin{tabular}{cccccc}
\hline$M_{s}^{t}(\beta)$ & $J_{I A E r}$ & $J_{I A E r}(\%)$ & $T V_{u r}$ & $T V_{u r}(\%)$ & $M_{s}^{r}$ \\
\hline $2.0(0.667)$ & 0.363 & $100 \%$ & 0.398 & $81 \%$ & 2.009 \\
$2.0(1.0)$ & 0.363 & $100 \%$ & 0.489 & $100 \%$ & 2.009 \\
\hline $1.2(2.501)$ & 0.471 & $66 \%$ & 0.201 & $101 \%$ & 1.239 \\
$1.2(1.0)$ & 0.717 & $100 \%$ & 0.200 & $100 \%$ & 1.239 \\
\hline
\end{tabular}

\section{CONCLUSIONS}

The use of a Two-Degree-of-Freedom (2-DoF) PID controller must allow the control-loop designer to take into consideration the regulatory control performance and control effort requirements in conjunction with the control system robustness and then improve the servo-control performance.

However the analysis of the recommended tuning for its proportional set-point weight has shown that the established constraint by controller's manufactures for its values to the $0 \leq \beta \leq 1.0$ range, avoids the designer to exploit the full potential of these controllers.

The allowed range for the proportional set-point weight could make sense when the controller design main objectives were only to optimize its performance but nowadays, the performance-robustness trade-off is taken into account within the modern control design formulations. Even included explicitly into the tuning equations as it has been shown in the concrete tuning rules analyzed in this paper. It has been shown that this constraint reduces the performance of the control-loop responses, to a setpoint step change, when a high robustness control system is required.

The control system designer will be able to use the full inherent capabilities of the Two-Degree-of-Freedom PID controllers, only when the existing constraint in the setpoint weight selection will be removed by the manufacturers.

\section{ACKNOWLEDGEMENTS}

This work has received financial support from the Spanish CICYT program under grant DPI2007-63356.

The financial support from the University of Costa Rica and from the MICIT and CONICIT of the Government of the Republic of Costa Rica is greatly appreciated.

\section{REFERENCES}

ABB ((n.d.)). Protonic 100/500/550 Digitric 500 Configuration and Parametrization Manual. ABB Automation Products. 42/62-500 12EN.

Alfaro, V.M., Vilanova, R., and Arrieta, O. (2008). Analytical Robust Tuning of PI controllers for First-OrderPlus-Dead-Time Processes. In 13th IEEE International Conference on Emerging Technologies and Factory Automation. Hamburg-Germany.

Araki, M. (1984a). On Two-Degree-of-Freedom PID Control System. Technical report, SICE Research Commitee on Modeling and Control Design of Real Systems.

Araki, M. (1984b). PID Control Systems with Reference Feedforward (PID-FF Control System). In Proc. of 23rd SICE Anual Conference, 31-32.

Araki, M. (1985). Two-Degree-of-Freedom Control System - I. Systems and Control, 29, 649-656. 
Arrieta, O. and Vilanova, R. (2007a). Performance degradation analysis of Optimal PID settings and Servo/Regulation tradeoff tuning. CSCO\%, Conference on Systems and Control, Marrakech-Morocco.

Arrieta, O. and Vilanova, R. (2007b). PID Autotuning settings for balanced servo/regulation operation. MEDO\%, 15th IEEE Mediterranean Conference on Control and Automation, Athens-Greece.

Arrieta, O. and Vilanova, R. (2007c). Servo/Regulation tradeoff tuning of PID controllers with a robustness consideration. CDCO7, 46th IEEE Conference on Decision and Control, New Orleans, Louisiana-USA.

Arrieta, O., Vilanova, R., Alfaro, V., and Moreno, R. (2008). Considerations on PID Controller Operation: Application to a Continuous Stirred Tank Reactor. In 13th IEEE International Conference on Emerging Technologies and Factory Automation. Hamburg-Germany.

Åström, K. and Hägglund, T. (1995). PID Controllers: Theory, Design and Tuning. Instrument Society of America, Research Triangle Park, NC, USA.

Åström, K. and Hägglund, T. (2004). Revisiting the Ziegler-Nichols step respose method for PID control. Journal of Process Control, 14, 635-650.

Åström, K. and Hägglund, T. (2006). Advanced PID Control. ISA - The Instrumentation, Systems, and Automation Society.

Åström, K., Hang, C.C., Persson, P., and Ho, W.K. (1992). Towards Intelligent PID Control. Automatica, 28(1), 19.

Åström, K., Panagopoulos, H., and Hägglund, T. (1998). Design of PI controllers based on non-convex optimization. Automatica, 34, 585-601.

Babb, M. (1990). Pneumatic Instruments Gave Birth to Automatic Control. Control Engineering, 37(12), 20-22.

Bennett, S. (2000). The Past of PID Controllers. In IFAC Digital Control: Past, Present and Future of PID Control. Terrassa, Spain.

Chien, I., Hrones, J., and Reswick, J. (1952). On the automatic Control of generalized passive systems. Trans. ASME, 175-185.

Cohen, G.H. and Coon, G.A. (1953). Theoretical Considerations of Retarded Control. ASME Transactions, 75, Jul.

Emerson (2008). DeltaV BooksOnLine9.3. Emerson Process Managment. Web-based version http://www.easydeltav.com/BOL/.

Foxboro (1998). Instruction 762 CNA Single Station Micro Controller. The Foxboro Company. MI 018-885.

Fuji (2001). Instruction Manual - Compact Controller M. Fuji Electric. INP-TN1PDA 3cE.

Gorez, R. (2003). New desing relations for 2-DOF PID-like control systems. Automatica, 39, 901-908.

Hägglund, T. and Åström, K. (2002). Revisiting the Ziegler-Nichols tuning rules for PI control. Asian Journal of Control, 4, 354-380.

Hang, C. and Cao, L. (1996). Improvement of Transient Response by means of variable set point weighting. IEEE Transaction on Industrial Electronics, 4, 477-484.

Honeywell (2007). UDC 3500 Universal Digital Controller Product Manual. Honeywell International. 51-52-25-120.

Horowitz, I.M. (1963). Shynthesis of Feedback Systems. Academic Press.
Kaya, I. (2004). Tuning PI controllers for stable process with specifications on gain and phase margings. ISA Transactions, 43, 297-304.

López, A.M., Miller, J.A., Smith, C.L., and Murrill, P.W. (1967). Tuning Controllers with Error-Integral Criteria. Instrumentation Technology, 14, 57-62.

Martin, J., Smith, C.L., and Corripio, A.B. (1975). Controller Tuning from Simple Process Models. Instrumentation Technology, 22(12), 39-44.

Méndez, V. (2008). Performance and Robustness of PID Control Loops. Licenciatura Thesis, Escuela de Ingeniería Eléctrica, Universidad de Costa Rica. (in Spanish).

Mitsubishi (2002). System Q Programmable Controller Logic Control Programming Manual. Mitsibishi Electric. 149256.

O'Dwyer, A. (2003). Handbook of PI and PID Controller Tuning Rules. Imperial College Press, London, UK.

Omron (2007). Instructions Reference Manual Programmable Controllers SYSMAC CS Series. Omron Electronics LLC.

Rivera, D.E., Morari, M., and Skogestad, S. (1986). Internal Model Control. 4. PID Controller Desing. Ind. Eng. Chem. Des. Dev., 25, 252-265.

Rockwell (2003). MicroLogix 1200 and 1500 Programmable Controllers Bulletin 1762 and 1764 Instruction Set Reference Manual. Rockwell Automation.

Rockwell (2005). Logix 5000 Controllers Process Control and Drivers Instructions User Manual. Rockwell Automation. PN 957955.

Rovira, A., Murrill, P.W., and Smith, C.L. (1969). Tuning Controllers for Setpoint Changes. Instrumentation \& Control Systems, 42, 67-69.

Sung, S.W. and Lee, I.B. (1999). PID Controllers and Automatic Tuning. A-JIN Publishing Co., Seul, Korea.

Taguchi, H. and Araki, M. (2000). Two-Degree-ofFreedom PID controllers - Their functions and optimal tuning. In IFAC Digital Control: Past, Present and Future of PID Control. Terrassa, Spain.

Taguchi, H. and Araki, M. (2002). Survey of researches on Two-Degree-of-Freedom PID controllers. In The 4 th Asian Control Conference. Singapore.

Taguchi, H., Kokawa, M., and Araki, M. (2002). Optimal tuning of two-degree-of-freedom PD controllers. In The 4th Asian Control Conference. Singapore.

Visioli, A. (2006). Practical PID Control. Springer Verlag Advances in Industrial Control Series.

Yokogawa ((n.d.)). YS 1500 Indicating Controller User Manual. Yokogawa Electric Company. IM 01B08B01$02 \mathrm{E}$

Ziegler, J.G. and Nichols, N.B. (1942). Optimum Settings for Automatic Controllers. ASME Transactions, 64, $759-768$. 